\title{
Ramification has little impact on shoot hydraulic efficiency in the sexually dimorphic genus Leucadendron (Proteaceae)
}

\author{
Adam Roddy ${ }^{\text {Corresp., }}$, Justin J van Blerk ${ }^{2}$, Jeremy J Midgley ${ }^{2}$, Adam G West ${ }^{2}$ \\ ${ }^{1}$ Department of Integrative Biology, University of California, Berkeley, Berkeley, CA, United States of America \\ 2 Biological Sciences, University of Cape Town, Cape Town, South Africa \\ Corresponding Author: Adam Roddy \\ Email address: adam.roddy@berkeley.edu
}

Despite the diversity of branching architectures in plants, how this morphological variation influences hydraulic efficiency has been poorly studied. Branch junctions are commonly thought to be points of high hydraulic resistance, but adjustments in leaf area or xylem conduit abundance or dimensions could compensate for the additional hydraulic resistance of nodal junctions at the level of the entire shoot. Here we used the sexually dimorphic genus Leucadendron (Proteaceae) to test whether variation in branch ramification impacts shoot hydraulic efficiency. We found that branch ramification was related to leaf traits via Corner's rules such that more highly ramified shoots had smaller leaves, but that branch ramification had little consistent impact on shoot hydraulic efficiency, whether measured on a leaf area or stem cross-sectional area basis. These results suggest that the presumed increase in resistance associated with branching nodes can be compensated by other adjustments at the shoot level (e.g. leaf area adjustments, increased ramification to add additional branches in parallel rather than in series) that maintain hydraulic efficiency at the level of the entire shoot. Despite large morphological differences between males and females in the genus Leucadendron, which are due to differences in pollination and reproduction between the sexes, the physiological differences between males and females are minimal. 
1 Ramification has little impact on shoot hydraulic efficiency in the sexually dimorphic genus Leucadendron (Proteaceae)

3 Adam B. Roddy ${ }^{1,2}$, Justin J. van Blerk ${ }^{3}$, Jeremy J. Midgley ${ }^{3}$ and Adam G. West ${ }^{3}$

$4 \quad{ }^{1}$ Department of Integrative Biology, University of California, Berkeley, Berkeley, CA, USA

$5 \quad{ }^{2}$ Current address: School of Forestry \& Environmental Studies, Yale University, New Haven,

6 CT, USA ${ }^{3}$ Department of Biological Sciences, University of Cape Town, Cape Town, South

7 Africa

8

9 Author for correspondence:

10 Adam B. Roddy

11 adam.roddy@berkeley.edu

12 phone: +510.224 .4432$

13 


\section{Abstract}

16 Despite the diversity of branching architectures in plants, how this morphological variation

17 influences hydraulic efficiency has been poorly studied. Branch junctions are commonly

18 thought to be points of high hydraulic resistance, but adjustments in leaf area or xylem

19 conduit abundance or dimensions could compensate for the additional hydraulic resistance

20 of nodal junctions at the level of the entire shoot. Here we used the sexually dimorphic

21 genus Leucadendron (Proteaceae) to test whether variation in branch ramification impacts

22 shoot hydraulic efficiency. We found that branch ramification was related to leaf traits via

23 Corner's rules such that more highly ramified shoots had smaller leaves, but that branch

24 ramification had little consistent impact on shoot hydraulic efficiency, whether measured

25 on a leaf area or stem cross-sectional area basis. These results suggest that the presumed

26 increase in resistance associated with branching nodes can be compensated by other

27 adjustments at the shoot level (e.g. leaf area adjustments, increased ramification to add

28 additional branches in parallel rather than in series) that maintain hydraulic efficiency at

29 the level of the entire shoot. Despite large morphological differences between males and

30 females in the genus Leucadendron, which are due to differences in pollination and

31 reproduction between the sexes, the physiological differences between males and females

32 are minimal. 
34

35

36

37

38

39

40

41

42

43

44

45

46

47

48

49

50

51

52

53

54

55

56

57

58

59

60

61

62

\section{Introduction}

Plants are remarkably diverse in gross morphology. Theoretical studies have shown that the number of optimal plant architectures increases with the number of functions that must be performed (Niklas 1994). One notable trait that varies both within and among species is the degree of branch ramification. Because branches and stems serve both hydraulic and biomechanical functions, larger leaves and leaf areas are correlated with thicker branches (Corner 1949; Ackerly \& Donoghue 1998; Olson et al. 2009). According to Corner's rules, branch size is inversely related to how highly ramified shoots are; that is, given a constant allocation of biomass, the stems of more highly branched shoots should, on average, be smaller. Furthermore, branch size and ramification may also be linked to reproductive characters: in species that bear flowers on terminal shoots, selection on inflorescence size and number is associated with leaf size via the allometric scaling of each of them with stem size (Bond \& Midgley 1988; Midgley \& Bond 1989). Corner's rules has, therefore, provided a useful framework for studying plant architecture (Lauri 2018). Subsequent theory sought to explain constancy in the relationship between leaf area and stem size with a hydraulic argument (Shinozaki et al. 1964), although the underlying assumption of the pipe model theory that all xylem in the stem are conductive is not valid (Lehnebach et al. 2018). One open question is, therefore, whether variation in stem size and branch ramification affects hydraulic efficiency: are more highly ramified shoots with smaller branches less hydraulically efficient? If more higlight ramified branches are less hydraulically efficient, then branching would limit plant architectural variability and potentially trade-off with production of terminal structures (e.g. leaves and inflorescences).

Studies that have mapped the hydraulic architecture of trees have found large variability in leaf-specific stem hydraulic conductivity (i.e. the hydraulic conductivity normalized to the supplied leaf area) throughout the crowns of adult trees (Tyree et al. 1983; Ewers \& Zimmermann 1984b a; Tyree \& Ewers 1991; Tyree \& Alexander 1993; Tyree \& Zimmermann 2002). Stem segments immediately basal to branch junctions tend to have higher conductivity than the junctions themselves (Ewers \& Zimmermann 1984a b), although this difference is not as great as the variation in leaf specific conductivity 
63 throughout the rest of the crown (Tyree \& Alexander 1993). Nonetheless, branch junctions

64 are typically thought to increase hydraulic resistance (Tyree \& Zimmermann 2002),

65 meaning that more highly branched shoots may be less conductive. However, because most

66 stem hydraulic measurements are made on unbranching stem segments, they typically

67 include multiple years of growth and do not include the terminal branches where conduits

68 taper dramatically (Anfodillo et al. 2013; Olson et al. 2014). Furthermore, how conductivity

69 of a stem segment-or loss of conductivity due to embolism in the stem segment-influences

70 the hydraulic conductance of the entire shoot network is not immediately intuitive. How

71 resistance (the reciprocal of conductance) is partitioned throughout the plant will dictate

72 how conductivity of a stem segment scales with shoot conductance (Meinzer 2002;

73 Brodersen et al. 2019). The implication is that there can be significant declines in

74 conductivity of a stem segment (due to either embolism formation or to branch junctions)

75 without impacting the hydraulic conductance of the entire shoot.

76 Here we tested whether branch ramification reduces shoot hydraulic conductance. We 77 compared males and females of the genus Leucadendron (Proteaceae). Leucadendron is 78 known for its high variability in sexual dimorphism, with males and females of some 79 species being highly dimorphic while males and females of other species exhibit little 80 dimorphism. Leucadendron species display a full range of phenotypes-from

81 monomorphism to dimorphism-in leaf size, branch size and ramification, and inflorescence 82 size, with males generally having smaller, more numerous leaves and branches (Williams

83 1972; Bond \& Midgley 1988; Midgley \& Bond 1989). The most striking differences between

84 males and females of dimorphic Leucadendron species are differences in leaf size and

85 branch ramification (Figure 1). Theoretical and empirical studies in other systems have

86 long considered leaf size to be mechanistically linked to branch size because of the

87 biomechanical constraints on the distribution of leaves (Corner 1949; Ackerly \& Donoghue

88 1998; Olson et al. 2009). In dimorphic Leucadendron species, females have larger leaves

89 and thicker, less ramified stems than their conspecific males. Furthermore, females of many

90 Leucadendron species maintain their cones in the canopy for several years, a strategy

91 termed 'serotiny'. Serotiny allows seeds to be dispersed only after fire, but preventing the

92 cones from opening during the hot, dry Mediterranean summers requires a continuous 
93 supply of water and carbon. Although the water costs of maintaining cones are lower than

94 originally thought (Cramer \& Midgley 2009), the relative costs of reproduction are

95 assumed to be higher for females than for males (Barrett \& Hough 2013 but see Bond \&

96 Maze (1999)), and these costs are further expected to increase with increasing serotiny. It

97 was recently proposed that these high female costs may select for higher hydraulic

98 efficiency in less ramified females (Harris \& Pannell 2010). However, this has yet to be

99 directly tested, and the limited available data have found either no difference in hydraulic

100 conductivity between sexes or males with higher conductivity (Jacobsen et al. 2007), and

101 no difference in water use efficiency between males and females (Midgley 2010).

102 We compared two co-occurring Leucadendron species: L. daphnoides is monomorphic with

103 males and females that have similar degrees of ramification and leaf size, while L. rubrum is

104 extremely dimorphic with males being more highly ramified with smaller leaves than

105 females (Figure 1). First, we developed a metric for quantifying branch ramification that is

106 applicable beyond just Leucadendron and show that this metric of ramification scales

107 predictably with traits linked to Corner's rules. Second, we measured whole-shoot

108 hydraulic conductance for shoots ranging in size for all four species-by-sex combinations.

109 Our analyses showed that more ramified branches (either between sexes or between

110 species) were no less hydraulically efficient, challenging the assumption that higher

111 ramification results in a lower hydraulic efficiency.

\section{Methods}

\section{Plant material}

114 Males and females of both species grow naturally near Du Toitskloof Pass, Western Cape,

115 South Africa. All individuals were co-occurring within $\sim 10 \mathrm{~m}$ of each other on a slope

116 extending westward toward the city of Paarl. The two species differ in the degree of

117 serotiny, with L. rubrum holding its cones for an average of 2.8 years and L. daphnoides for

118 less than one year (Williams 1972; Harris \& Pannell 2010). Plants were collected and

119 measurements made during November-December 2012 and during April-May 2013. In the

120 field, mature plants were chosen, ignoring any unusually small individuals. Shoots were cut 
121 at the plant base with garden shears and immediately recut under water one node above

122 the previous cut. Individual shoots were placed in dark, plastic bags, and their bases

123 submerged in water during transport back to the lab. Shoots were stored in a $4^{\circ} \mathrm{C}$

124 refrigerator until the day of measurement, and any shoots not measured within three days

125 of field collection were discarded.

126

127

128

129

130

131

132

133

134

135

136

137

138

139

140

141 $142\left(K_{C S A}\right)$.

\section{Measurement of hydraulic conductance}

Immediately prior to hydraulic measurements, shoots were defoliated underwater. When necessary, a sharp blade was used to cut leaves at the petiole base. Stems were recut underwater with a new, carbon steel blade. Hydraulic conductance was measured on whole, branching shoots of various sizes using a low pressure flow meter (Kolb et al. 1996). With this method, the cut stem base was inserted into a compression fitting (Omnifit) that was connected to hard-sided tubing, the opposite end of which was submerged in a vial of filtered $0.01 \mathrm{M} \mathrm{KCl}$ that sat on a balance (Mettler-Toledo MS205DU with a resolution of $0.01 \mathrm{mg}$ ). The branching shoot was then placed inside a chamber connected to a vacuum pump. Flow rates from the balance were recorded automatically every 5-20 seconds (the frequency depended on the absolute flow rate) at each of a series of pressures below ambient: 10, 30, 50, 65, 40, $20 \mathrm{kPa}$. The stable flow rate at each pressure was determined when the coefficient of variation of the previous ten instantaneous flow rates was less than $5 \%$. Hydraulic conductance, $K$, was obtained by a linear regression of flow rate as a function of pressure $\left(\mathrm{kg} \mathrm{s}^{-1} \mathrm{MPa}^{-1}\right)$. To compare hydraulic efficiency between groups, $K$ was normalized by either entire shoot leaf area $\left(K_{L A}\right)$ or by cross-sectional area of the stem base

\section{Quantifying ramification and other traits}

144 At the end of each measurement, shoots were assessed for other morphological traits. The 145 cross-sectional area of the stem base was calculated from the average of two perpendicular 146 measurements of stem diameter at the base using manual calipers. Shoot ramification was 147 quantified in two ways. First, we quantified the rate of diameter change down the length of 148 a subset of the shoots, using the method of Harris and Pannell (2010) and described briefly

149 here. Starting at the highest branch, we measured stem cross-sectional area at the midpoint 
150 of each consecutive internode down the shoot as well as the relative position of this

151 midpoint along the length of the shoot. The slope of the relationship between the logarithm

152 of stem cross-sectional area and the relative distance from the crown provides an index of

153 ramification. In using this method to quantify ramification, Harris and Pannell (2010)

154 measured the tallest shoot on a plant and measured nodes all the way to the base of the

155 plant. However, because we sought to directly link shoot remification to hydraulic

156 conductance and wanted to capture a wide range of shoot sizes, the shoots we measured

157 were not as large as those measured previously by Harris and Pannell (2010). Nonetheless,

158 because their method for quantifying ramification depends on a linear regression,

159 removing the basalmost nodes should not unduly impact estimates of ramification so long

160 as the assumptions of linear regression are met. Furthermore, the method of quantifying

161 ramification used by Harris and Pannell (2010) assumes that all branches arise

162 immediately below terminal inflorescences and at nodes. For some species of

163 Leucadendron, including L. daphnoides, this assumption is valid. However, in other species,

164 particularly males of L. rubrum, most branches occur along the internodes. To account for

165 these many small branches we measured the total number of branch tips on a shoot

166 (regardless of their size and position on the shoot) normalized by the cross-sectional area

167 of the stem base. The number of branch tips per stem cross-sectional area (BTSA) can

168 easily be applied to shoots of any size, including shoot segments from the most recent year

169 of growth that do not have properly defined internodes, as well as to other species with

170 different branching patterns.

171 Leaves were placed on a flatbed scanner and their total area and number determined per

172 shoot using ImageJ (Schneider et al. 2012). The average leaf size per shoot (termed here

173 simply 'leaf size') was determined by dividing the total leaf area by the total number of

174 leaves on the shoot. For consistency with Corner's rules, we quantified the average leaf

175 area per branch by dividing the total shoot leaf area by the total number of terminal

176 branches on each shoot. 


\section{Data analysis}

178 All data were analyzed using R v. 3.0.2 (Wickham 2017; R Core Team 2018). Scaling

179 relationships were determined using standard major axis regression (SMA) as

180 implemented in the 'smatr' package (Warton et al. 2012). For each pair of variables,

181 relationships were determined for each species and sex combination, and differences

182 between groups in scaling relationships determined using a likelihood ratio test as

183 implemented in the sma function. For certain scaling relationships, slope tests were used to

184 test whether slopes significantly differed from unity. If the likelihood ratio test (LRT)

185 revealed no significant differences in scaling slopes between groups, data were pooled and

186 the relevant slopes and statistics shown and reported. We also compared $K_{L A}$ and $K_{C S A}$

187 between groups using ANOVA with species, sex, and the interaction between them as

188 factors. If either of these grouping variables were significant, post-hoc Tukey HSD tests

189 were used to determine which groups were significantly different. For visualization

190 purposes, 95\% confidence intervals around scaling slopes were estimated by bootstrap

191 sampling 1000 iterations.

192 Results

193

Stem and leaf architectural relationships

194 Stems with larger basal cross-sectional areas held more leaf area (Figure 2). There was no

195 significant difference in slopes between sex or species groups ( $\mathrm{LRT}=4.146, \mathrm{df}=3, \mathrm{P}=$

196 0.246), and the global slope was significantly greater than unity (slope $=1.11, R^{2}=0.83, P<$

197 0.001).

198 The two metrics of branch ramification did not covary linearly, even in log-log space

199 (Figure S1). Subsequent analyses focused solely on BTSA because it can be readily applied

200 to any branching architecture and it conformed to Corner's rules (Figure 3). More highly

201 ramified branches held significantly less leaf area per branch tip (slope $=-0.97, R^{2}=0.93, P$

$202<0.001$; slope test: $r=-0.10, \mathrm{df}=65, \mathrm{P}=0.41$ ) with no significant difference between

203 groups (LRT $=1.50, \mathrm{df}=3, P=0.68$; Figure 3a). Similarly, more highly ramified shoots had

204 smaller leaves on average (Figure 3b). Although species exhibited different elevations in 
205 their relationships, there was no significant difference in the slopes among groups (LRT = $2062.74, \mathrm{df}=3, P=0.43 ;$ L. rubrum: slope $=-0.39, R^{2}=0.87, P<0.001 ;$ L. daphnoides: slope $=-$ $\left.2070.45, R^{2}=0.46, P<0.001\right)$.

208 To determine whether larger branches were more hydraulically efficient, we tested 209 whether there were different scaling slopes between $K$ and two metrics of shoot size: total 210 leaf area and basal cross-sectional area (Figure 4). There was no significant differences in 211 slopes between groups for the scaling of $K$ and leaf area ( $L R T=6.19, \mathrm{df}=3, \mathrm{P}=0.10$ ), and 212 the slope test revealed that the scaling slope was not significantly different from unity $(r=$ $2130.0097, \mathrm{df}=66, P=0.94$; Figure $4 \mathrm{a}$ ). Thus, total shoot leaf area was a strong predictor of 214 whole-shoot hydraulic conductance across species and sexes $\left(R^{2}=0.83, P<0.001\right)$.

215 Similarly, there was no significant difference between groups in the scaling slopes between $216 K$ and stem cross-sectional area ( $\mathrm{LRT}=5.93, \mathrm{df}=3, \mathrm{P}=0.12$ ), and there was no significant 217 difference between the observed slope and unity $(r=0.212, \mathrm{df}=58, \mathrm{P}=0.11$; Figure $4 \mathrm{~b})$.

218 Thus, basal cross-sectional area was a strong predictor of whole shoot hydraulic 219 conductance across species and sexes $\left(R^{2}=0.69, P<0.001\right)$.

220 To determine whether ramification impacted hydraulic efficiency, we tested whether there 221 was a significant relationship between BTSA and leaf area-specific hydraulic conductance $222\left(K_{L A}\right)$ and between BTSA and hydraulic conductance $(K)$ normalized by stem basal cross223 sectional $\left(K_{C S A}\right)$. There was no universal effect of BTSA on either $K_{C S A}(P=0.79$; Figure 5a) 224 or $K_{L A}(P=0.13$, Figure $5 b)$. Within certain species and sex groups, however, there were 225 some significant effects of BTSA. Only in L. daphnoides females did more highly ramified 226 individuals have lower $K_{C S A}\left(R^{2}=0.30, P=0.02\right.$; Figure 5a). In contrast, more ramified $L$. 227 rubrum males had higher $K_{C S A}\left(R^{2}=0.42, P<0.02\right.$; Figure $\left.5 a\right)$. There was no significant of 228 species, sex, or the interaction between species and sex on $K_{C S A}$ in an ANOVA (all $P>0.05$ ). 229 Similarly, only for L. daphnoides females was $K_{L A}$ lower for more ramified individuals $\left(R^{2}=\right.$ $230 \quad 0.36, P=0.01$; Figure $5 \mathrm{~b}$ ), and when L. rubrum males and females were pooled together 231 there was a weak, but significant negative relationship between BTSA and $K_{L A}\left(R^{2}=0.16, P\right.$ $232=0.02$; Figure 5b). ANOVA revealed a similar pattern, with a significant effect of the 233 interaction of species and $\operatorname{sex}(F=4.59, \mathrm{df}=1, P=0.04)$ on $K_{L A}$. However Tukey post-hoc 234 HSD test found no single pairwise comparison significant, even before Bonferroni 
235 adjustment, at the $\mathrm{P}=0.05$ level. The largest pairwise difference was between $L$. rubrum

236 males and females, although this difference was not significant in either the Tukey post-hoc

237 comparison $(P=0.14)$ or in a Welch's two-sample t-test $(t=1.997, \mathrm{df}=18.55, P=0.06)$.

238 Discussion

239 In the present analysis of two co-occurring Leucadendron species differing in their degrees

240 of sexual dimorphism, we found no consistent support for hydraulic differences between

241 the sexes, or even between species. Despite substantial differences in gross morphology,

242 there was little effect of changes in leaf or stem morphology on hydraulic conductance and

243 hydraulic efficiency. Overall, these results suggest that while Corner's rules dictate the

244 relationships between leaf size, inflorescence size and number, and branch ramification,

245 there is little net effect of these traits on the hydraulic functioning of the stem. Previous

246 work has argued that in Leucadendron the accumulation of resistance in branch junctions

247 and the requirement for serotinous females to supply water to long-lived seed cones would

248 drive sexual dimorphism in branch ramification (Harris \& Pannell 2010), but our results do

249 not support the underlying assumptions of these ideas.

250 Allometry of leaves and stems obeys Corner's rules

251 Corner's rules predict that "the stouter the stem, the bigger the leaves and the more

252 complicated their form" (Corner 1949). Corner's first prediction between stem size and leaf

253 size has been well-characterized for many species including Leucadendron (Bond \&

254 Midgley 1988; Ackerly \& Donoghue 1998; Olson et al. 2009). Across species and sexes a

255 single slope explained the relationship between total shoot leaf area and stem cross-

256 sectional area with larger stems holding proportional more leaf area (i.e. SMA slope not

257 significantly different from unity; Figure 2). This relationship further suggests that despite

258 large variation in stem diameter both within and among species, leaf area is allocated

259 proportional to stem diameter.

260 More apropos to the present study, Corner also predicted that "the greater the ramification, 261 the smaller become the branches and their appendages" (Corner 1949). This prediction 262 emerges out of an argument of allocation: given a constant amount of biomass, producing 
263 more branches means that each of them must be smaller. In most species of Leucadendron,

264 branches emerge just below terminal inflorescences and seed cones. This branching

265 pattern results in internodal segments bracketed by nodes from which a variable number

266 of branches emerge. In dimorphic Leucadendron, males have more branches at each node

267 than their conspecific females, which is driven by pollinator selection for more

268 inflorescences because inflorescences are borne only on terminal branches (Bond \& Maze

269 1999). Selection on inflorescence number, therefore, can result in more highly ramified

270 branches and, because of Corner's rules, smaller leaves [Midgley:1989aa]. Using BTSA as a

271 simple metric of ramification, we found that more highly ramified shoots both within and

272 among species and sexes bore less leaf area per branch, driven predominantly by each leaf

273 being smaller (Figure 3). These results were consistent with Corner's second prediction,

274 with isometric scaling between BTSA and leaf area per branch tip. Interestingly, while

275 leaves were smaller on more ramified shoots, there was a species-specific effect such that

276 the two species had different intercepts (Figure 3b). In contrast, another metric of

277 ramification recently used in a broad survey of Leucadendron species (Harris \& Pannell

278 2010) exhibited no similar scaling relationships with leaf size or with leaf area per tip

279 (Figure S2).

\section{Little effect of shoot morphology on shoot hydraulics}

281 We sought to test whether these large differences in leaves and stems among species and

282 sexes in Leucadendron led to differences in stem hydraulic efficiency, as has been suggested

283 (Harris \& Pannell 2010). Two main characteristics of woody shoots were thought to be

284 important: the cross-sectional area of a branch and the number of branching nodes. These

285 two traits are, to some extent, linked in Leucadendron because more highly ramified shoots

286 are more likely to have more nodes and smaller individual branches. As a rough

287 approximation, larger diameter branches should have higher conductance because they

288 have more xylem conduits (assuming no change in conduit size with branch size). However,

289 wood is complex, being composed of vessels, fibers, parenchyma, and pith in various

290 proportions (Zanne et al. 2010), such that hydraulic conductance does not necessarily scale

291 isometrically with cross-sectional area. The complexity of wood structure-function

292 relationships means that simplistic models that treat the stem as a pipe cannot accurately 
293 describe shoot water transport capacity (Lehnebach et al. 2018). For example, because

294 stems continually grow, their theoretical hydraulic capacity always increases, yet as xylem

295 ages it becomes progressively non-functional, such that most of the water transpired by

296 leaves is delivered via the current year's xylem (Melcher et al. 2003). As a result, cross-

297 sectional area of the stem cannot necessarily predict the hydraulic capacity of the stem.

298 Despite numerous measurements on the hydraulic conductivity of stems, surprisingly few

299 studies have examined the hydraulic conductance or conductivity of branch junctions

300 (nodes). The available data suggest that branch junctions increase hydraulic resistance, but

301 the magnitude of this effect is highly species specific (Ewers \& Zimmermann 1984b a;

302 Ewers et al. 1989; Tyree \& Ewers 1991; Tyree \& Alexander 1993). In Leucadendron, most

303 species produce new branches each year almost exclusively just below terminal

304 inflorescences and, in the case of females, seed cones. Leaves are borne on newer branches,

305 and so transpired water must traverse multiple nodal junctions before it reaches the

306 leaves. Yet, because the effect of junctions on hydraulic resistance is species-specific,

307 simply having more nodes does not necessarily translate into higher resistance.

308 We found no compelling evidence that hydraulic efficiency differs between conspecific

309 males and females or even between the co-occurring species we studied here, which differ

310 dramatically in their degrees of sexual dimorphism. At the entire shoot level, ramification

311 had little impact on hydraulic efficiency, whether determined on a leaf area basis or on a

312 stem cross-sectional area basis (Figure 5). Among L. daphnoides females more highly

313 ramified individuals had lower $K_{C S A}$, but L. rubrum males-which were the most highly

314 ramified shoots we measured-exhibited the opposite relationship. When measured on a

315 leaf area basis, hydraulic efficiency was lower in more highly ramified L. daphnoides

316 females and among L. rubrum, but only when pooling males and females together. Overall,

317 these results suggest that ramification per se has little impact on hydraulic efficiency at the

318 level of the entire shoot. While branch junctions may add resistance to the shoot, the

319 amount of resistance is likely small and compensated for by other adjustments, such as

320 changes in leaf area or additional xylem area produced in basal segments. Furthermore,

321 assuming a given length of stem growth, adding this stem as an additional branch rather

322 than by growing the length of an existing branch would actually be beneficial because 
323 resistances are additive in series and conductances are additive in parallel. Thus, adding a

324 stem length as a branch in parallel would add to the conductance of the entire shoot rather

325 than subtract from it, as adding that stem length in series would. This feature of resistance

326 networks could compensate for the resistance of adding a branch node, and other studies

327 have suggested that trees may become more ramified as they grow taller (i.e. they add

328 parallel conductors) in order to compensate for the added resistance a longer path length

329 (Mencuccini \& Grace 1996). The similar water use efficiencies of Leucadendron males and

330 females supports the equivalence of their hydraulic efficiencies (Midgley 2010). Thus,

331 larger stems, whether males or females, provide no more water to their transpiring leaves

332 per unit leaf area or stem cross-sectional area than do smaller stems, in contrast to some

333 arguments about how differences in hydraulic efficiency between males and females may

334 be linked to sexual dimorphism in the genus (Harris \& Pannell 2010).

335 Males and females of each species must survive to the next fire events in order to be 336 represented in the most recent year's seed crop and, thus, maximize fitness (Midgley

337 2000). Conspecific males and females should, therefore, have similar longevities and likely

338 also similar rates of metabolism. This is supported by the similarity in hydraulic efficiencies

339 among conspecifics we studied and the strong correspondence between shoot hydraulic

340 efficiency and photosynthetic capacity (Brodribb \& Feild 2000; Brodribb et al. 2002). If the

341 hydraulic architecture of females were more efficient than that of males, then it is not clear

342 why males would not also have evolved a similar branching and leaf structure given that

343 males and females co-occur and may compete with each other. Also, if the hydraulic

344 architecture of males were less efficient, then males may incur costs associated with

345 reproduction equal to or higher than those of females, contrary to most theory and data

346 regarding the costs of reproduction (Bond \& Maze 1999). Instead, our data and those of

347 Midgley (2010) are consistent with there being similar hydraulic efficiencies between

348 species and sexes, regardless of the degrees of sexual dimorphism and branch ramification.

\section{Conclusions}

350 Despite substantial variation between species and sexes in morphological traits of leaves

351 and stems, these differences did not translate into differences in hydraulic efficiency. More 
352 highly ramified individuals had smaller leaves, consistent with Corner's rules, but there 353 were no consistent effects of these morphological modifications on hydraulic efficiency. 354 These results suggest that selection may act on males and females in Leucadendron to 355 maintain physiological performance while allowing for large variation in morphological 356 traits that could be under divergent selection. These results further highlight the 357 importance of recasting studies of hydraulic efficiency at the level of the entire shoot by 358 quantifying the pathways of water transport all the way to the leaves.

359

360 Acknowledgments

361 ABR was supported by Graduate Research Fellowship from the U.S. National Science 362 Foundation. 


\section{Figure legends}

366 Figure 1. Representative leaves with areas approximating the average leaf area for each 367 group. From left to right: L. daphnoides female and male, L. rubrum female and male.

368 Figure 2. Relationship between total shoot leaf area and stem cross-sectional area. A single 369 standard major axis regression described the scaling relationship across species and sexes 370 and the slope did not significantly differ from unity.

371 Figure 3. Corner's rules and the relationships between branch ramification (BTSA), leaf 372 size, and leaf area per branch tip. Solid lines indicate 1:1 relationship, and dashed lines 373 indicate the standard major axis regressions, with black lines indicating across all data and 374 colored lines indicating only within specific groups. (a) Relationship between leaf area per 375 tip and BTSA was explained by a single scaling relationship, whose slope was not 376 significantly different from isometry. (b) Relationship between average leaf size and BTSA 377 was highly significant within each species, and the two slopes were not significantly 378 different from each other.

379 Figure 4. Relationships between whole shoot hydraulic conductance $(K)$ and (a) shoot leaf 380 area and (b) cross-sectional area of the stem base. There was no significant difference 381 between sexes or species in these scaling slopes, and a single scaling relationship applied to 382 all data. Grey shading indicated 95\% confidence intervals around the slope of the 383 relationship. Solid lines indicate the 1:1 relationship, and dashed lines indicate the 384 standard major axis regression.

385 Figure 5. Shoot ramification had little impact on shoot hydraulic efficiency. (a) Shoot 386 hydraulic conductance per unit cross-sectional stem area as a function of BTSA. (c) Shoot 387 hydraulic conductance per unit leaf area as a function of BTSA. Solid colored lines indicates 388 a significant relationship within a sex x species combination, and dashed colored lines 389 indicate a significant relationship within a species $(P<0.01)$. 
390 Figure S1. Relationship between the two metrics of ramification: that used by Harris and 391 Pannell (2010; 'HP ramification') and that used in the present study (BTSA). Only within $L$. 392 daphnoides was there a correlation between the two metrics of ramification.

393 Figure S2. Relationships between the metric of ramification used by Harris and Pannell 394 (2010) (termed here 'HP ramification') and (a) the average leaf area per branch tip and (b) 395 the average leaf size. There was a significant relationship between HP ramification and the 396 leaf area per branch tip only for $L$. daphnoides $\left(R^{2}=0.43, P=0.05\right)$. No other relationships 397 were significant, despite the expected scaling relationship between ramification and leaf 398 size according to Corner's rules (Corner 1949). 
400 References

401 Ackerly D. \& Donoghue M. (1998) Leaf size, sapling allometry, and Corner's rules:

$402 \quad$ Phylogeny and correlated evolution in maples (Acer). The American Naturalist 152, 403 767-791.

404 Anfodillo T., Petit G. \& Crivellaro A. (2013) Axial conduit widening in woody species: A still 405 neglected anatomical pattern. IAWA Journal 34, 352-364.

406 Barrett S.C.H. \& Hough J. (2013) Sexual dimorphism in flowering plants. Journal of $407 \quad$ Experimental Botany 64, 67-82.

408 Bond W. \& Midgley J. (1988) Allometry and sexual differences in leaf size. The American $409 \quad$ Naturalist 131, 901-910.

410

411

412

413 414

415

416

417

418 419

420 421

422 423

424 425

Bond W.J. \& Maze K.E. (1999) Survival costs and reproductive benefits of floral display in a sexually dimorphic dioecious shrub, Leucadendron xanthoconus. Evolutionary Ecology 13, 1-18.

Brodersen C.R., Roddy A.B., Wason J.W. \& McElrone A.J. (2019) Functional status of xylem through time. Annual Review of Plant Biology In press.

Brodribb T.J. \& Feild T.S. (2000) Stem hydraulic supply is linked to leaf photosynthetic capacity: Evidence from New Caledonian and Tasmanian rainforests. Plant, Cell \& Environment 23, 1381-1388.

Brodribb T.J., Holbrook N.M. \& Gutiérrez M.V. (2002) Hydraulic and photosynthetic coordination in seasonally dry tropical forest trees. Plant, Cell \& Environment 25.

Corner E.J.H. (1949) The durian theory or the origin of the modern tree. Annals of Botany 13, 367-414.

Cramer M.D. \& Midgley J.J. (2009) Maintenance costs of serotiny do not explain weak serotiny. Austral Ecology 34, 653-662.

Ewers F. \& Zimmermann M. (1984a) The hydraulic architecture of balsam fir (Abies balsamea). Physiologia Plantarum 60, 453-458. 
426 Ewers F.W. \& Zimmermann M.H. (1984b) The hydraulic architecture of eastern hemlock 427 (Tsuga canadensis). Canadian Journal of Botany 62, 940-946.

428 Ewers F.W., Fisher J.B. \& Chiu S.-T. (1989) Water transport in the liana Bauhinia 429 fassoglensis (Fabaceae). Plant Physiology 91, 1625-1631.

430 431

432 433

434

435

436 437 438

439 440

441

442

443 444

445 446

447

448 449 450

Harris M.S. \& Pannell J.R. (2010) Canopy seed storage is associated with sexual dimorphism in the woody dioecious genus Leucadendron. Journal of Ecology 98, 509-515.

Jacobsen A.L., Agenbag L., Esler K.J., Pratt R.B., Ewers F.W. \& Davis S.D. (2007) Xylem density, biomechanics and anatomical traits correlate with water stress in 17 evergreen shrub species of the Mediterranean-type climate region of south africa. Journal of Ecology 95, 171-183.

Kolb K.J., Sperry J.S. \& Lamont B.B. (1996) A method for measuring xylem hydraulic conductance and embolism in entire root and shoot systems. Journal of Experimental Botany 47, 1805-1810.

Lauri P.-É. (2018) Corner's rules as a framework for plant morphology, architecture and functioning-issues and steps forward. New Phytologist 221, 1679-1684.

Lehnebach R., Beyer R., Letort V. \& Heuret P. (2018) The pipe model theory half a century on: a review. Annals of Botany 121, 773-795.

Meinzer F.C. (2002) Co-ordination of vapour and liquid phase water transport properties in plants. Plant, Cell \& Environment 25, 265-274.

Melcher P.J., Zwieniecki M.A. \& Holbrook N.M. (2003) Vulnerability of xylem vessels to cavitation in sugar maple. Scaling from individual vessels to whole branches. Plant Physiol 131, 1775-80.

Mencuccini M. \& Grace J. (1996) Developmental patterns of above-ground hydraulic conductance in a scots pine (Pinus sylvestris L.) age sequence. Plant, Cell \& Environment 19, 939-948. 
451 Midgley J. (2010) Causes of secondary sexual differences in plants - evidence from

452

453

454 455

456 457

458

459

460

461

462

463

464

465

466

467

468

469

470

471

472

473

474

475

476

extreme leaf dimorphism in leucadendron (proteaceae). South African Journal of Botany 76, 588-592.

Midgley J. \& Bond W.J. (1989) Leaf size and inflorescence size may be allometrically related traits. Oecologia 78, 427-429.

Midgley J.J. (2000) What are the relative costs, limits and correlates of increased degree of serotiny? Austral Ecology 25, 65-68.

Niklas K.J. (1994) Morphological evolution through complex domains of fitness.

Proceedings of the National Academy of Sciences 91, 6772-6779.

Olson M.E., Aguirre-Hernández R. \& Rosell J.A. (2009) Universal foliage-stem scaling across environments and species in dicot trees: Plasticity, biomechanics and corner's rules. Ecology Letters 12, 210-219.

Olson M.E., Anfodillo T., Rosell J.A., Petit G., Crivellaro A., Isnard S., ... Castorena M. (2014) Universal hydraulics of the flowering plants: Vessel diameter scales with stem length across angiosperm lineages, habits and climates. Ecology Letters 17, 988997.

Schneider C.A., Rasband W.S. \& Eliceiri K.W. (2012) NIH image to imagej: 25 years of image analysis. Nature methods $\mathbf{9 , 6 7 1 . ~}$

Shinozaki K., Yoda K., Hozumi K. \& Kira T. (1964) A quantitative analysis of plant form-the pipe model theory: I. basic analyses. Japanese Journal of ecology 14, 97-105.

R Core Team. (2018) R: A language and environment for statistical computing. R Foundation for Statistical Computing, Vienna, Austria.

Tyree M., Graham M., Cooper K. \& Bazos L. (1983) The hydraulic architecture of thuja occidentalis. Canadian Journal of Botany 61, 2105-2111.

Tyree M.T. \& Alexander J.D. (1993) Hydraulic conductivity of branch junctions in three temperate tree species. Trees-Structure and Function 7, 156-159.

PeerJ reviewing PDF | (2018:05:28297:1:0:NEW 28 Feb 2019) 
477 Tyree M.T. \& Ewers F.W. (1991) The hydraulic architecture of trees and other woody $478 \quad$ plants. New Phytologist 119, 345-360.

479 Tyree M.T. \& Zimmermann M.H. (2002) Xylem structure and the ascent of sap. Springer, $480 \quad$ Berlin.

481 Warton D.I., Duursma R.A., Falster D.S. \& Taskinen S. (2012) Smatr 3-an R package for 482 estimation and inference about allometric lines. Methods in Ecology and Evolution 3, 483 257-259.

484 Wickham H. (2017) Tidyverse: Easily install and load the 'tidyverse'.

485 Williams J.M. (1972) A revision of the genus leucadendron (proteaceae). The Bolus 486 Herbarium, University of Cape Town.

487 Zanne A.E., Westoby M., Falster D.S., Ackerly D.D., Loarie S.R., Arnold S.E.J. \& Coomes D.A. 488 (2010) Angiosperm wood structure: Global patterns in vessel anatomy and their 489 relation to wood density and potential conductivity. American Journal of Botany 97, 490 207-215. 


\section{Figure 1}

Representative leaves highlighting sexual dimorphism

Representative leaves with areas approximating the average leaf area for each group. From left to right: L. daphnoides female and male, L. rubrum female and male.
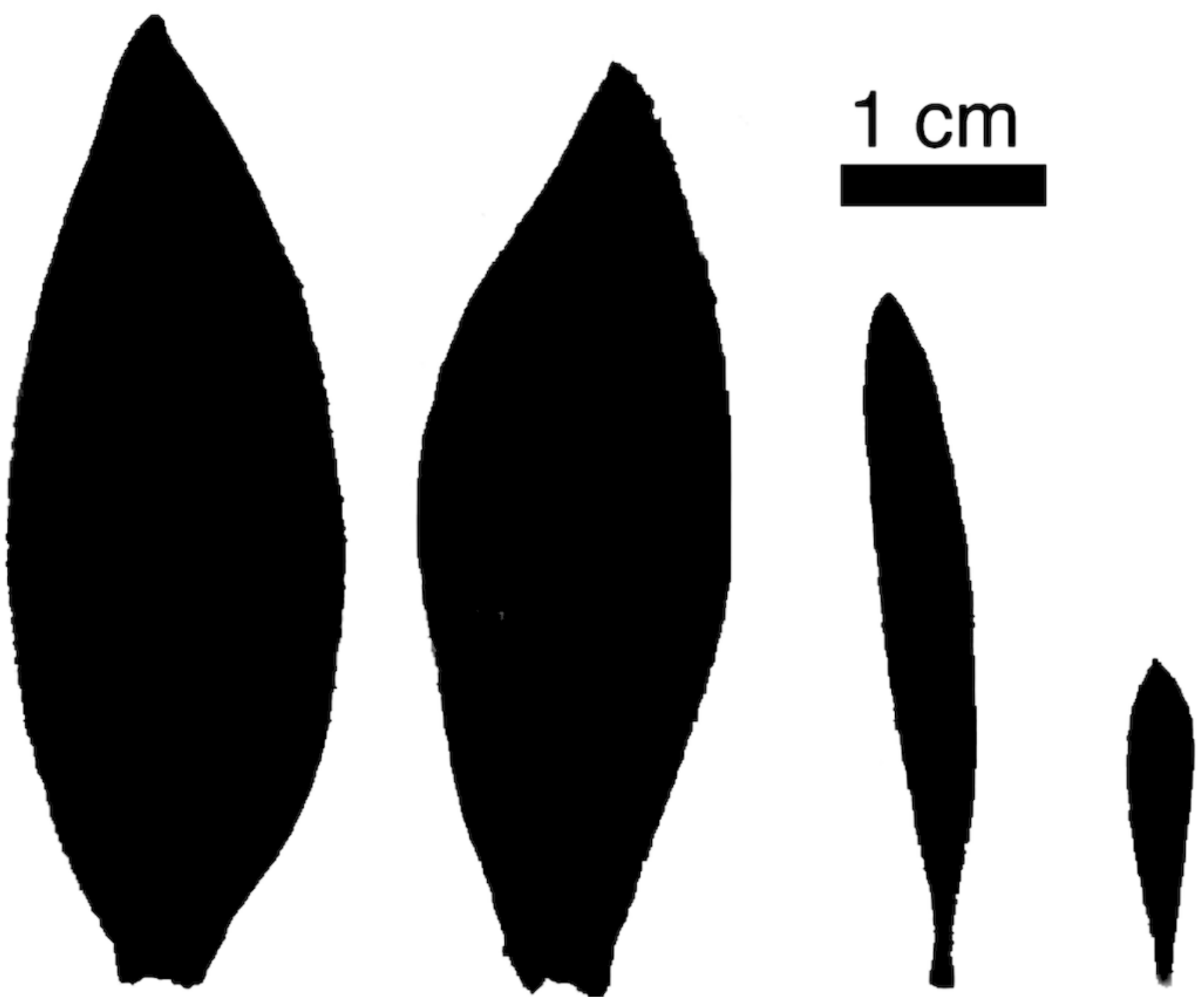
Figure 2 (on next page)

Relationship between total shoot leaf area and stem cross-sectional area.

A single standard major axis regression described the scaling relationship across species and sexes and the slope did not significantly differ from unity. 
Figure 3 (on next page)

Corner's rules and the relationships between branch ramification (BTSA), leaf size, and leaf area per branch tip.

Solid lines indicate 1:1 relationship, and dashed lines indicate the standard major axis regressions, with black lines indicating across all data and colored lines indicating only within specific groups. (a) Relationship between leaf area per tip and BTSA was explained by a single scaling relationship, whose slope was not significantly different from isometry. (b) Relationship between average leaf size and BTSA was highly significant within each species, and the two slopes were not significantly different from each other. 
PeerJ

Manuscript to be reviewed
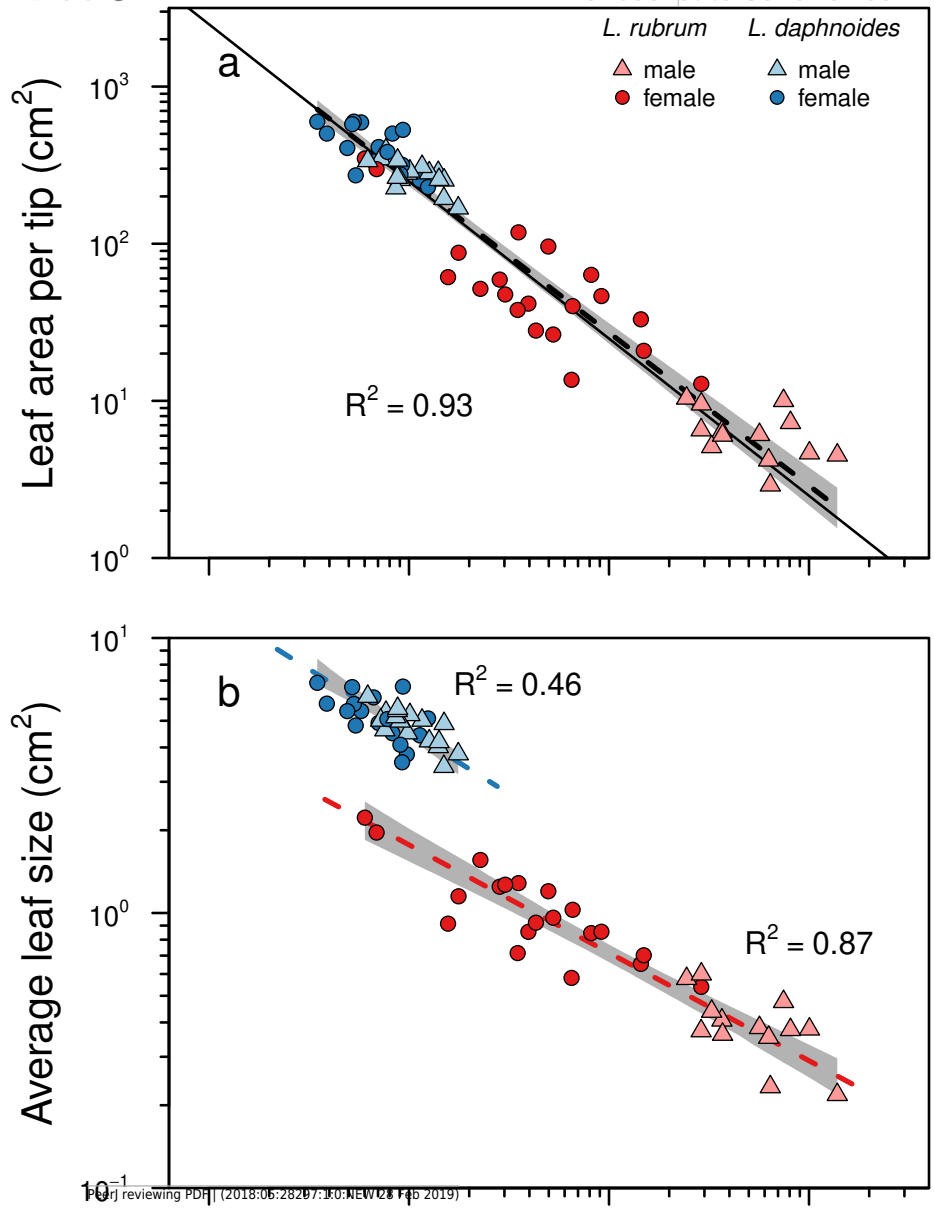

$10^{4}$

$10^{5}$

$10^{6}$

$10^{7}$

BTSA (no. tips $\mathrm{m}^{-2}$ ) 
Figure 4 (on next page)

Relationships between whole shoot hydraulic conductance and shoot size.

Relationships between whole shoot hydraulic conductance $(K)$ and $(a)$ shoot leaf area and (b) cross-sectional area of the stem base. There was no significant difference between sexes or species in these scaling slopes, and a single scaling relationship applied to all data. Grey shading indicated $95 \%$ confidence intervals around the slope of the relationship. Solid lines indicate the 1:1 relationship, and dashed lines indicate the standard major axis regression. 


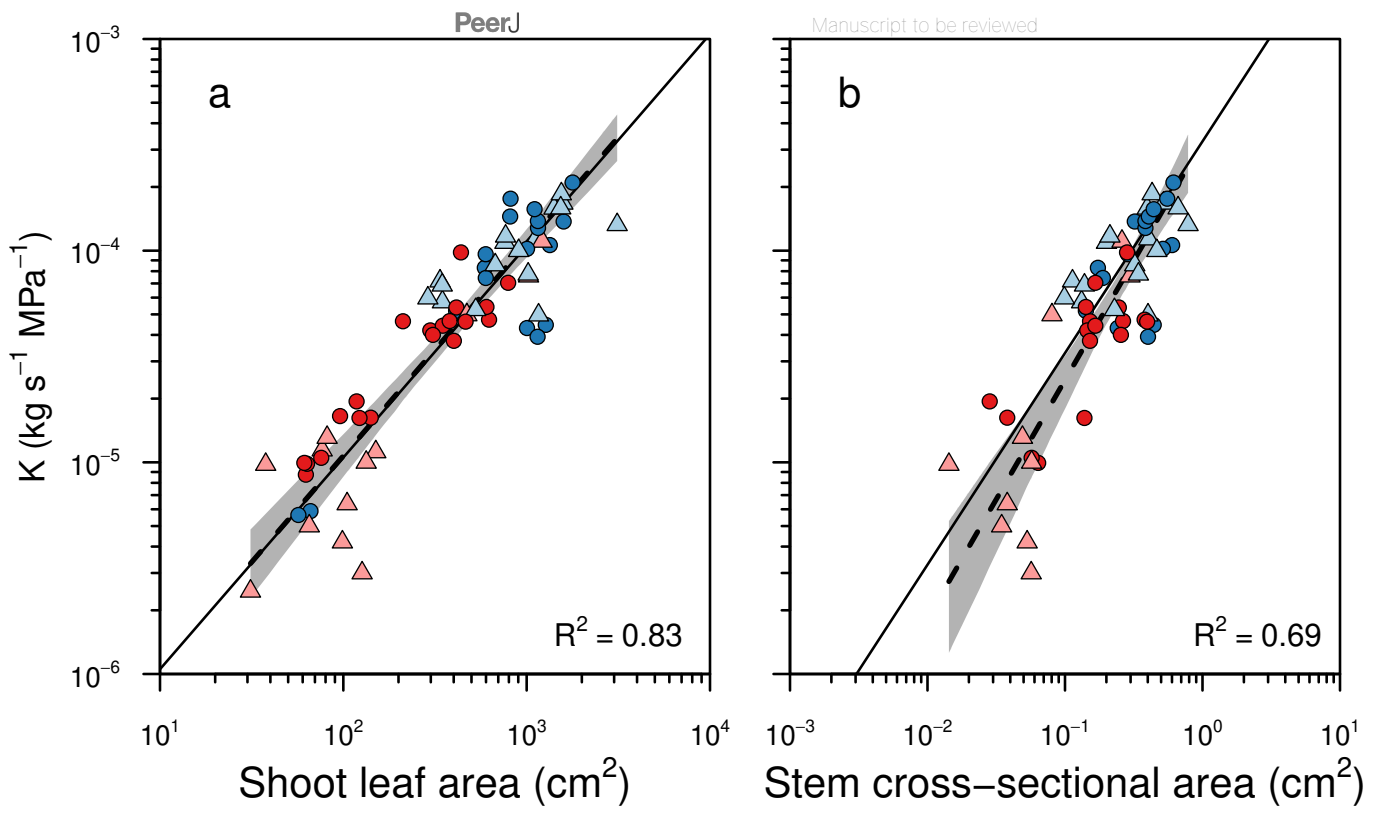


Figure $\mathbf{5}$ (on next page)

Shoot ramification had little impact on shoot hydraulic efficiency.

(a) Shoot hydraulic conductance per unit cross-sectional stem area as a function of BTSA. (c) Shoot hydraulic conductance per unit leaf area as a function of BTSA. Solid colored lines indicates a significant relationship within a sex $\mathrm{x}$ species combination, and dashed colored lines indicate a significant relationship within a species $(P<0.01)$. 

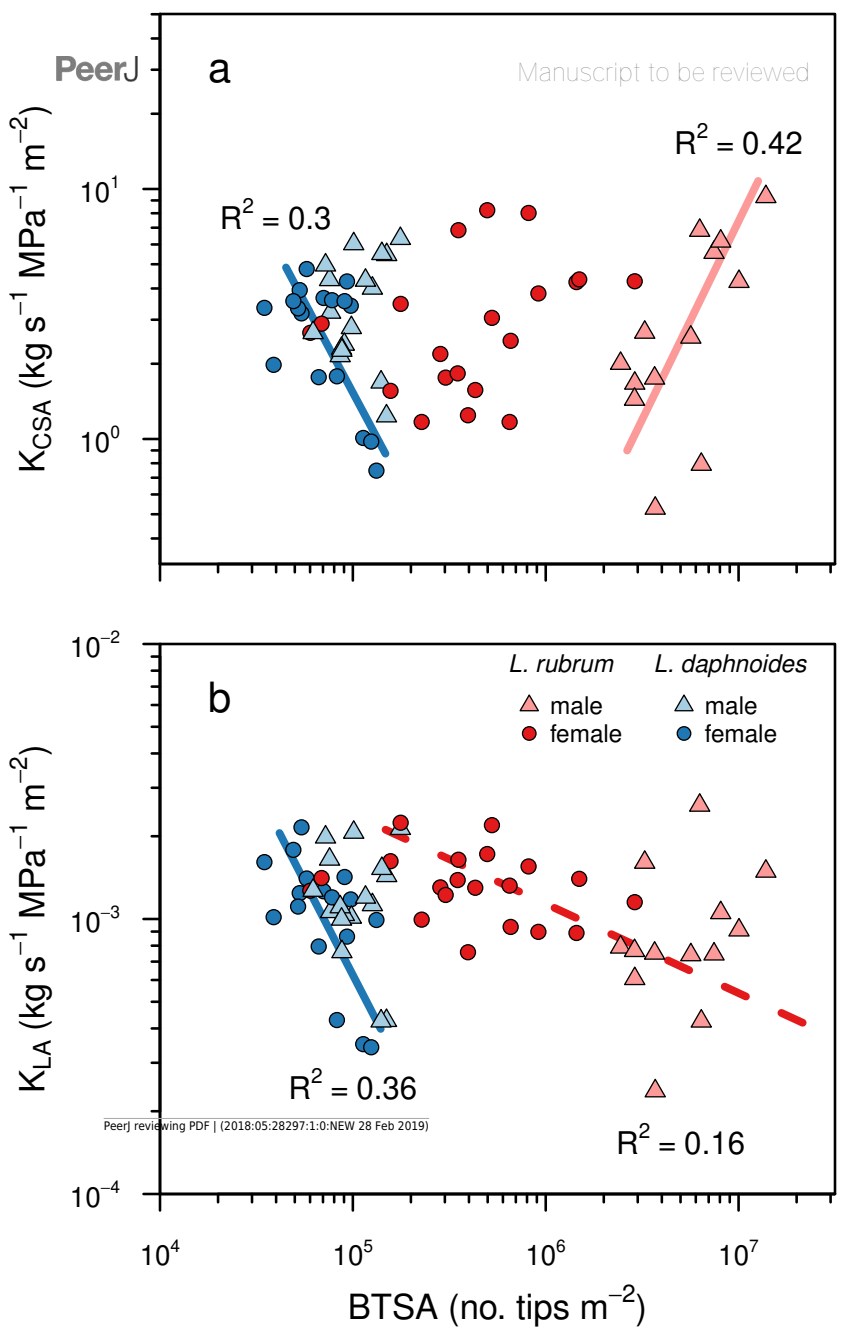